\title{
The Simulation Design of the Microstrip Antenna Based on The New Left-Handed Structure
}

\author{
Zhiliang Song \\ Department of information network \\ Dalian Air Force Communication NCO Academy \\ Dalian, China \\ zhiliang404@sohu.com \\ Rongtao Gao \\ Department of foreign languages \\ Hebei North University, \\ Zhangjiakou, China \\ anngrt@gmail.com
}

\author{
Chuanfang Bai \\ PLA Unit 61413, \\ Xiangfan, 441000, China \\ bigboy@189.cn \\ Xianfeng Zeng \\ Department of wire communication \\ Dalian Air Force Communication NCO Academy \\ Dalian, China \\ 20905247@bit.edu.cn
}

\begin{abstract}
It is a review of the distinct physical characteristics of left-handed materials. A new idea is proposed that the lefthanded materials are applied to the design of microstrip antenna. Two microstrip antennas are designed based on the new left-handed Structure which definitely enhance the directivity and widen the bandwidth of the antenna.
\end{abstract}

Keywords-Left-handed structure; Microstrip antenna; Simulation design

\section{INTRODUCTION}

In 1968, V. G. Veselago, a physicist from Soviet Union, predicted that in LHM, the direction of the phase transmission and direction of the energy transmission were opposite. Besides, when refraction rate was negative, the direction of the wave vector was opposite to the direction of energy transmission. $E, H$ and $k$ observe the left-hand law. That was the prototype of the LHM [1]. It was until 1996 that J.B.Pendry and other physicists from Britain who adopted periodical metal array and split ring resonators (SRR) realized the negative dielectric constant and the magnetic permeability [2]. Scientists created undiscovered maters by adopted the smart man-made structures. Those two structures drew quite a lot of attentions of the researchers from different fields. In the recent years, the LHM has become a new conception of material-design, and has been used in the design of micro strip antenna [3]. By using its extraordinary physical properties, the performance of the antenna will be greatly improved.

\section{EXTRAORDINARY ElECtromagnetic Properties OF LHM}

\section{A. Negative refraction property}

When the electromagnetic wave goes through the interface of normal media and the LHM, the "negative refraction" comes out. Generally, when the incident light goes through media 1 towards media 2, the refractions comes out, as is shown in Figure 1. According to the Snell principle, the refraction angle $\theta_{2}$ and the incident angle $\theta_{1}$ satisfy the equation:

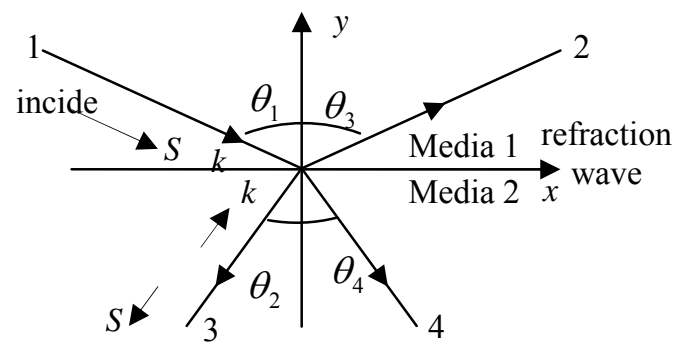

Figure 1. Reflection and refraction of the electromagnetic wave on the interface with positive and negative refraction rate

$$
n_{1} \sin \theta_{1}=n_{2} \sin \theta_{2}
$$

in equation ( 1$), n_{1}, n_{2}$ are the refraction rates. Refraction rate can be described as the equation $n^{2}=\varepsilon \mu$. If it is the traditional materials $(\mu>0, \varepsilon>0)$, the refraction rate $n=\sqrt{\varepsilon \mu}$, is positive. If media 2 is the LHM ( $\mu<0, \varepsilon<0)$, the result will be different. When the incident wave goes through media 1 towards media 2 , the boundary conditions should be fulfilled according to the Maxwell equations:

$$
\begin{gathered}
E_{t 1}=E_{t 2} \\
H_{t 1}=H_{t 2} \\
\varepsilon_{1} E_{n 1}=\varepsilon_{2} E_{n 2} \\
\mu_{1} H_{n 1}=\mu_{2} H_{n 2}
\end{gathered}
$$

If media 1 is the traditional material and media 2 is the LHM, i.e. $\varepsilon_{1}>0, \mu_{1}>0, \varepsilon_{2}<0, \mu_{2}<0, \boldsymbol{E}$ and $\boldsymbol{H}$ have the same direction in the horizontal direction, while the two have 
the opposite directions in the vertical direction, and within the media 2, k, $\boldsymbol{E}$ and $\boldsymbol{H}$ comply with the left-hand rule. At the moment, a different kind of refraction occurs: the refraction wave and the incident wave are on the same side of the normal of the interface. The direction of the energy flow $\mathrm{S}$ of the refracted light is opposite to that of vector $k$. If we define the refraction rate $n_{2}$ of media 2 is negative as

$$
n_{2}=-\sqrt{\varepsilon_{2} \mu_{2}}
$$

then $\theta_{2}$ is negative compared with normal refraction angle, when the Snell principle is still tenable. Thus the LHM is also called "negative index of refraction (NIR)" media.

\section{B. Reversed Cerenkov radiation}

The charging particles moving in a constant speed in the vacuum do not radiate electromagnetic wave. But when charged particles moving in a constant speed in media, they will generate the induced current and therefore form a series of second wave source to generate the second wave. When the velocity of the particles exceeds the light speed in the media, the second waves interfere with each other and then radiate the electromagnetic waves. This is called the Cerenkov radiation. In traditional media, the wave front generated after the interference is a cone as is shown in Figure 2(a). The energy of the electromagnetic wave is transmitted along the norm of the cone. The direction of the energy radiation and the moving direction of the particles form an angle $\theta . \theta$ is defined by equation(7):

$$
\cos \theta=\frac{c}{n v}
$$

$V$ represents the velocity of the moving particles. But in the LHM, the direction of the energy transmission is opposite to that of the initial velocity. Therefore, the direction of the energy radiation is opposite to the direction of the moving particle. The two directions form a forward cone angle as is shown in Figure 2(b). This is the inverse Cerenkov radiation [4].

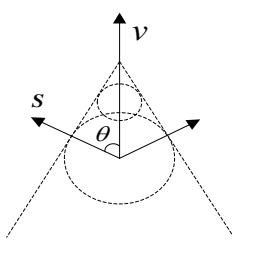

(a)

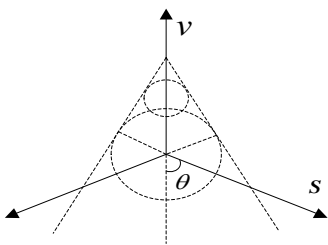

(b)
Figure 2. Cerenkov radiation $(V$ is the direction of the moving particle, $S$ is the direction of the power, and $\theta$ is the included angle)

\section{Reversed Doppler effect}

When the electromagnetic wave with the frequency of $\omega_{0}$ at the source transmits through the traditional media, if the detector is approaching the light source, the detected frequency is higher than $\omega_{0}$. Otherwise, the detected frequency is lower than $\omega_{0}$. That's what we call Doppler
Effect in the transmission of electromagnet. In LHM, the direction of the phase velocity and the direction of the group velocity are opposite, i.e. the directions between power transmitting and the phase transmitting are opposite. Thus, when the detector is approaching to the signal source, the detected frequency becomes lower, otherwise, the detected frequency becomes higher.

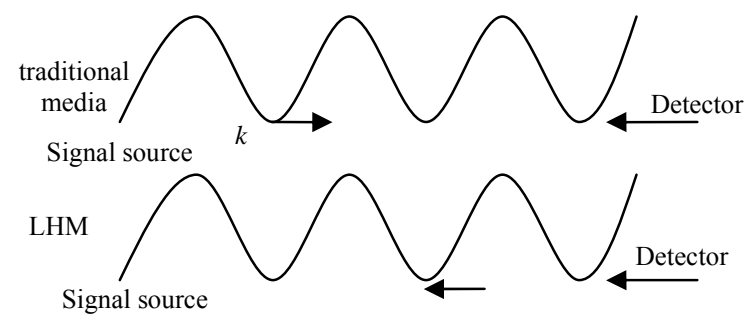

Figure 3. Doppler Effect in two different kinds of media

\section{SimUlation DESIGN OF THE LEFT-HANDED STRUCTURE UNIT}

\section{A. Extraction of constructive parameters of structure}

The constructive parameters of the media are the equivalent electric constant and the equivalent magnetic permeability. With the constructive parameters of the LHM media, we can describe the electromagnetic characteristics of the existing LHM; evaluate the performance and the range of frequency, all of which would show the extraordinary electromagnetic characteristics. That is significant in the structure of the design of LHM and optimizing its electromagnetic performances.

Among those experiments of extraction of constructive parameters of structure, the S-parameter method proposed by Smith gets the exact electromagnetic parameters of the LHM, and with those parameters one can calculate the equivalent electric constant and the equivalent magnetic permeability in a simple and practice way. Thus his method draws quite lots of attention [5-7].

Suppose that some plane wave incidences a plane with the thickness of $d$, which is made of LHM, the scattering parameters are $S_{11}$ and $S_{21}$. The refraction rate $n$ and the impedance $z$ fulfill the equation (1) with the scattering parameters [8].

$$
\begin{aligned}
S_{11} & =\frac{R_{01}\left(1-e^{j 2 n k_{0} d}\right)}{1-R_{01}^{2} e^{j 2 n k_{0} d}} \\
& S_{21}=\frac{\left(1-R_{01}^{2}\right) e^{j 2 n k_{0} d}}{1-R_{01}^{2} e^{j 2 n k_{0} d}} \\
\text { in which } & R_{01}=\frac{Z-1}{Z+1}
\end{aligned}
$$

the inverses of equations (8) and (9) are:

$$
\begin{aligned}
Z & = \pm \sqrt{\frac{\left(1+S_{11}\right)^{2}-S_{21}^{2}}{\left(1-S_{11}\right)^{2}-S_{21}^{2}}} \\
e^{j 2 n k_{0} d} & =X \pm j \sqrt{1-X^{2}}
\end{aligned}
$$


in which $\quad X=\frac{1}{2 S_{21}}\left(1-S_{11}^{2}+S_{21}^{2}\right)$

to the extraction of constructive parameters of structure of the passive LHM, equations (11) and (12) are determined by equations $Z^{\prime} \geq 0$ and $n^{\prime \prime} \geq 0$

Its refraction rate is

$$
n=\frac{1}{k_{0} d}\left\{\left[\ln \left(e^{j 2 n k_{0} d}\right)^{2}+2 m \pi\right]-j\left[\ln \left(e^{j 2 n k_{0} d}\right)^{\prime}\right]\right\}
$$

In the equation, $m=0, \pm 1, \pm 2, \cdots \cdots$ is the integer related to the real part of the refraction rate.

Thus, the S-parameter method not only draws the curves of the refraction rate and impedance, but also the equivalent electric constant and the equivalent magnetic permeability of the LHM [9].

\section{B. Simulation of the LHM structure unit}

In Smith's paper, he built the model of the LHM structure with the high-frequency electromagnetic simulation soft "Ansoft HFSS". The model is built up of the media plate with open resonant rings and slender metal bars on both sides.

The simulation demonstrates that around $10 \mathrm{GHz}$, the electric constant $\varepsilon$ of the material is negative; around 8$12.5 \mathrm{GHz}$ the magnetic permeability $\mu$ is negative as well. When the frequency approach $10 \mathrm{GHz}$, both the electric constant and the magnetic permeability reach negative, it's easy to deduce that the refraction rate is negative. The conclusion drawn by Smith leads the researchers to a new stage of the LHM. In this paper, a double-layer SRR structure of the LHM with its inner ring and outer ring connected is designed, and the performance of its parameters are simulated.

The double-layer SRR structure of the LHM with its inner ring and outer ring connected is shown as Figure 4. Suppose that the thickness of the media plate is $0.25 \mathrm{~mm}$, its width is $\mathrm{L}_{1}=2.5 \mathrm{~mm}, \mathrm{~L}_{2}=2.2 \mathrm{~mm}, \mathrm{~g}_{1}=0.3 \mathrm{~mm}, \mathrm{~g}_{2}=0.15 \mathrm{~mm}$, the simulation result is shown as in Figure 5-7. Its refraction rate, the equivalent electric constant and the equivalent magnetic permeability are negative within the range of $1.9-3.3 \mathrm{GHz}$ and 5.0-8.4GHz. It's obvious that with the same size, this kind of structure acquires lower resonant frequency. It can be adopted in the design of LF microwave devices.

\section{ApPlying the LHM in the Micro Strip ANTENNA DESIGN}

The extraordinary electromagnetic characters make the LHM widely adopted in the design of microwave antenna. Taking the advantages of negative refraction rates, one could use the LHM to fabricate the radome of the micro stripe antenna. It can restrain the edge radiation of the antenna effectively, promote the directivity of the antenna, and can restrain the edge scatterance. Thus the radiation efficiency will be promoted. With the conception of LHM characters, a micro stripe antenna with triple-ring SRR radome is proposed.
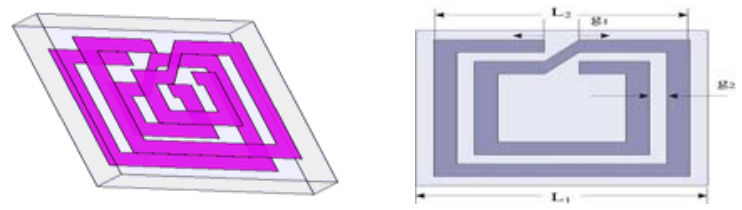

Figure 4. A double-layer SRR structure with its inner ring and outer ring connected

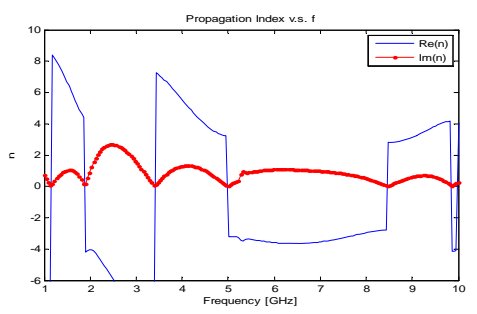

Figure 5. The refraction rate of the double-layer SRR structure with its inner ring and outer ring connected

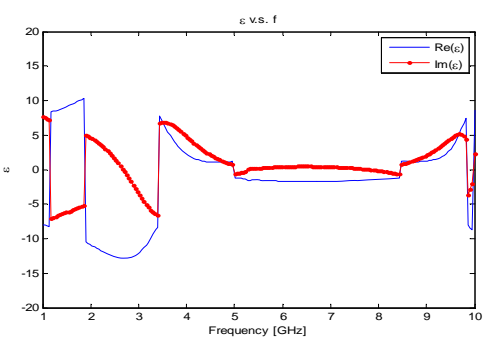

Figure 6. The equivalent electric constant of the structure

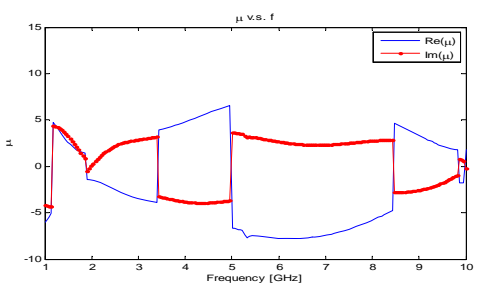

Figure 7. The equivalent magnetic permeability of the structure

The triple-ring SRR structure unit is shown as in Figure 9. Its size is $\mathrm{G} 1=0.5 \mathrm{~mm}, \mathrm{G} 2=1 \mathrm{~mm} . \mathrm{W} 1=1 \mathrm{~mm}, \mathrm{~W} 2=0.5 \mathrm{~mm}$, $\mathrm{L} 1=15.1 \mathrm{~mm}, \mathrm{~L} 2=9.1 \mathrm{~mm}, \mathrm{~L} 3=7.1 \mathrm{~mm}$, one can organize a left-handed structure array with this unit, as is shown in Figure. 10. The thickness of the media is $1.6 \mathrm{~mm}$, the electric constant is 2.6 , and the space between the units is $1 / 4$ wavelength of the central frequency.

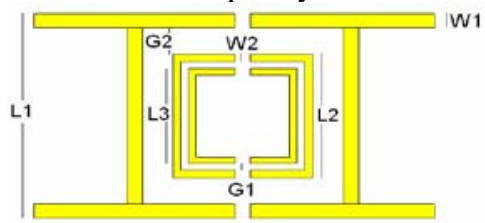

Figure 8. Triple-ring SRR structure unit (only the metal parts are visible) 


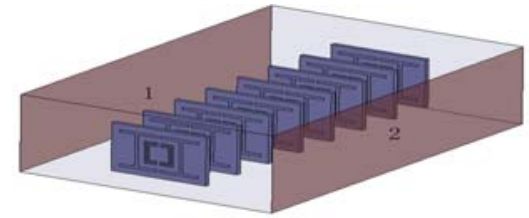

Figure 9. Triple-ring SRR structure array

The traditional micro stripe feeding antenna with surface mounted device(SMD) is as shown in Figure 10 (a). The length of the square SMD antenna is $40.5 \mathrm{~mm}$, the thickness of the media is $1.57 \mathrm{~mm}$, the width of the $50 \Omega$ feeder is $4.8 \mathrm{~mm}$, in the middle, the size of the impedance changer is $24.6 \mathrm{~mm} \times 0.8 \mathrm{~mm}$. We adopt the LHM on this antenna, as is shown in Figure 10 (b), the LHM is $13.5 \mathrm{~mm}$ away from the floor of the antenna, and the size of the antenna is $120 \mathrm{~mm} \times 120 \mathrm{~mm} \times 30.5 \mathrm{~mm}$. The return losses of the antenna with and without the LHM are shown in Figure 11; it's obvious that the bandwidth of the antenna with the LHM is much wider. Furthermore, we simulate the radiation pattern of the two antennas with the software CST; the results are shown in Figure 12-13. The maximum gain of the antenna has ascended from $7.47 \mathrm{~dB}$ to $11.5 \mathrm{~dB}$, the raise of the gain is significant.

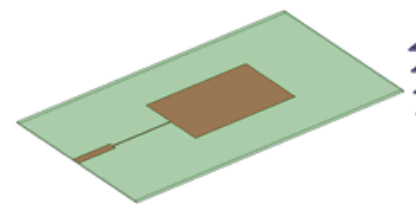

(a)

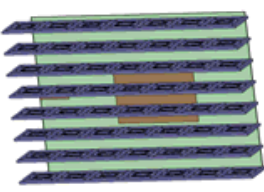

(b)
Figure 10. The micro stripe feeding antenna with LHM

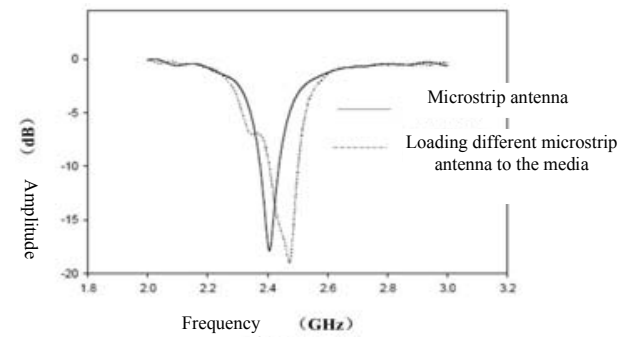

Figure 11. The return losses of the two antennas
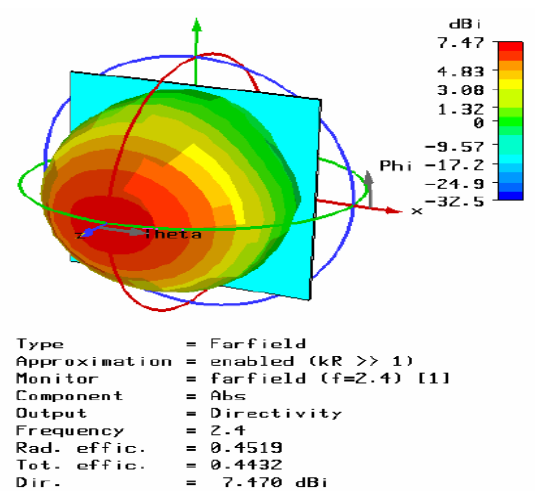

Figure 12. The radiation pattern of the traditional micro stripe antenna

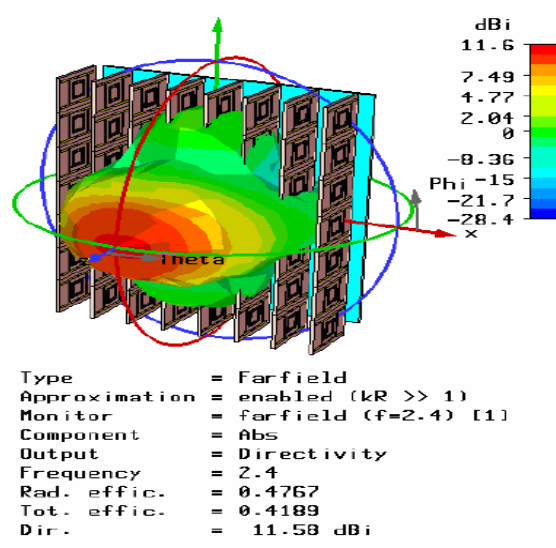

Figure 13. The radiation pattern of the micro stripe antenna with LHM

\section{CONCLUSION}

The extraordinary electromagnetic characters of the LHM confirm that it will be widely used in the wireless communication in the future. With further research, there will be more achievements and breakthroughs. Combining the LHM with the design of the micro stripe antenna has become a hot topic nowadays; it will influence the wireless communication in the future.

\section{REFERENCE}

[1] V G Veselago. The electrodynamics of substances with simultaneously negative values of $\varepsilon$ and $\mu[\mathrm{J}]$. Soviet Physics, January 1968 , vol.10,No.4: 509-514.

[2] Pendry J B, Holden A J, Stewart W J, et al. Extremely low frequency plasmons in metallic mesostructures[J].Phys.Rev.Lett.,1996,76(25): 4773-4776.

[3] Pendry J B, Holden A J, Robbins D J, et al. Magnetism from comductors and enhanced nonlinear phenomena[J]. IEEE Trans. Microwave Theory Tech., 1999, 47(11): 2075-2084.

[4] D Schurig, J J Mock, B J Justice, etal. Metamaterial electromagnetic cloak at microwave frequencies[J]. Science, 2006,314(5801): 977-980.

[5] Smith D R, Gollub J, Mock J J, Padilla W J and Schurig D. Calculation and Measurement of Bianisotropy in a Split Ring Resonator Metamaterial. Journal Of Applied Physics(J), 2006,100:02407:1-9.

[6] Ziolkowski R W, Kipple A D. Application of double negative materials to increase the power radiated by electrically small antennas[J]. IEEE Transactions on Antennas and Propagation. 2003, 51(10):2626-2640.

[7] Sanshui Xiao, Linfang Chen, Sailing He. A novel directional coupler utilizing a left-handed material[J]. IEEE Photonics Technology Letters. 2004, 16(1):171-173.

[8] Nicolson A M, Ross G F. Measurement of the intrinsic properties of materials by time-domain techniques[J]. IEEE Trans Instrum and Meas. 1970, 19:377-382.

[9] Pendry J B, Holden A J, Robbins D J, et al. Magnetism from conductors and enhanced nonlinear phenomena. IEEE Trans. Microwave Theory Tech.,1999, 47(11):2075-2084. 